application. Road kills have also been identified as a major threat to amphibian fauna. 7 out of 30 amphibian species which recorded in the Knuckles range are point endemic; but several previously unknown species await description. They would be at risk of extinction before they are even named. Threats to the unique recognized biodiversity \& the challenges to its conservation, demand urgent international \& national level scientific attention, policy \& planning. Therefore, it is our own responsibility to conserve our natural heritage without any delay.

\title{
V-01-9: Effects of Increased Salinity and an Introduced Predator on Lowland Amphibians in Southern China: Species Identity Matters
}

\author{
Nancy E. Karraker, James Arrigoni, David Dudgeon; University of Hong Kong
}

Approximately $30 \%$ of amphibian species are threatened due to a variety of factors affecting their habitats and physiology, yet contributions that interactions among factors make to population declines are not well-explored. Two factors, introduced mosquitofish (Gambusia affinis) and increased salinity, may coincide in lowland habitats used by breeding amphibians. Mosquitofish have been introduced worldwide and can be significant predators of amphibian larvae. Salinization of wetlands is an increasing problem globally due to (1) application of road deicing salts in temperate regions, (2) irrigation practices associated with intensive agriculture, particularly in Australia, and (3) saltwater intrusion due to sea-level rise. We investigated the effects of mosquitofish (Gambusia affinis) and increasing salinity on five species of lowland wetland-breeding amphibians from southern China. We exposed anuran hatchlings to four salinity levels and two fish treatments and all combinations of the two factors in a series of experiments. Four of the species were susceptible to predation by mosquitofish, two were sensitive to increased salinity at concentrations $>6 \%$ seawater, and one was tolerant of both increased salinity and mosquitofish. We found no interaction between the predator and increased salinity. Salinization and mosquitofish represent significant threats to lowland amphibians in this region and, coupled with the ongoing loss and degradation of lowland wetlands, portend a bleak future for lowland amphibian populations in the region.

\section{V-01-10: Attempt at Artificial Breeding and Analysis of Genetic Diversity in an Endangered Frog Odorrana ishikawae: Case Study of Fauna Conservation from Japan}

\author{
Masayuki Sumida; Inst. for Amphibian Biol., Hiroshima Univ.
}

Odorrana ishikawae, a species endemic to the Okinawa and Amami Islands, is described as the most beautiful frog in Japan. Over-hunting and environmental destruction over the last several decades have devastated the population of this species. Odorrana ishikawae has therefore been listed as class B1 endangered species in the IUCN Red List, and designated as a natural monument in both Okinawa and Kagoshima Prefectures. In the present study we: (1) artificially bred frogs and tested a farming technique in the laboratory to preserve this species; (2) analyzed mitochondrial DNA to evaluate the genetic diversity and the population composition of this species; and (3) extracted antimicrobial peptides from the skin to find biologically active agents with strong antimicrobial effect. The sample animals were artificially bred from four male/female pairs of Amami Island via artificial insemination. Among 3,078 eggs, 2,742 cleaved normally, and 1,761 metamorphosed normally. About 400 six-year-old mature frogs produced via artificial breeding are now being raised in the laboratory. During last year's breeding season, second-generation offspring were produced by natural mating activities of these frogs. The mitochondrial ND2 gene was found to be the most useful for elucidating genetic diversity and population composition of this species. The genetic diversity was relatively low (1.0\%) within the Okinawa and Amami populations, however, comparatively high (7.5\%) between the Okinawa and Amami populations. The Okinawa population was also differentiated from the Amami population both morphologically and reproductively. These populations can be appropriately regarded as different species. We succeeded in identifying 11 antimicrobial peptides from the skin of this endangered species. Some peptides showed strong antimicrobial activity and wide antimicrobial spectrum against bacteria. There is a chance that these peptides will contribute to the production of new antibiotics in the future. 